\title{
Measurements of ion energies in the tokamak plasma boundary
}

\author{
M. Kočan ${ }^{*, a, b}$, J. P. Gunn ${ }^{\text {, }}$, S. Carpentier-Chouchana ${ }^{c}$, A. Herrmann ${ }^{\mathrm{a}}$, A. Kirk ${ }^{\mathrm{d}}$, M. Komm ${ }^{\mathrm{e}}$,

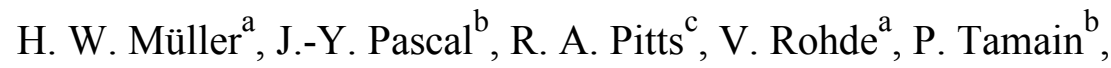 \\ ASDEX Upgrade and Tore Supra Teams \\ ${ }^{a}$ Max-Planck-Institut für Plasmaphysik, Bolzmannstr. 2, D-85748 Garching, Germany \\ ${ }^{b}$ CEA, IRFM, F-13108 Saint-Paul-lez-Durance, France \\ ${ }^{c}$ ITER Organization, Route de Vinon, CS 90 046, 13067 Saint Paul Lez Durance Cedex, France \\ ${ }^{d}$ EURATOM/CCFE Fusion Association, Culham Science Centre, Abingdon, Oxon OX14 3DB, UK \\ ${ }^{e}$ Association Euratom-IPP.CR, Institute of Plasma Physics, 18200 Prague Czech Republic
}

PACS: 52.40.Hf, 52.55.Fa

JNM keywords: Plasma-Materials Interaction

PSI-16 keywords: Edge plasma, First wall, Ion-surface Interactions, Probes

*Corresponding author address: Max-Planck-Institut für Plasmaphysik, Bolzmannstr. 2,

D-85748 Garching, Germany

*Corresponding author E-mail: martin.kocan@ipp.mpg.de

Presenting author: Martin Kočan

Presenting author e-mail: martin.kocan@ipp.mpg.de 


\begin{abstract}
Measurements of ion energies in the boundary of tokamak plasmas in L-mode discharges and during ELMs are reviewed. A profile of the ion-to-electron temperature ratio $T_{i} / T_{e}$ from the edge of the confined plasma into the scrape-off layer (SOL) is produced by compiling the available $\mathrm{T}_{\mathrm{i}}$ measurements. The picture that emerges is that in the SOL, as well as in the edge, $T_{i}$ is systematically higher than $T_{e}$ (ratios up to 10 just outside the last closed flux surface) for most plasma parameter regimes. Far SOL ELM ion energies measured in JET, and more recently in MAST and AUG, agree with the models of the ELM transients, providing strong evidence that ELM ions can reach the first wall with significant fraction of the pedestal energies.
\end{abstract}




\section{Introduction}

It has been evident for decades that the success of tokamaks as fusion reactors will be strongly influenced by plasma-wall interaction processes. Predictions of plasma-wall interactions in ITER rely on the experimental database from existing tokamaks. In the SOL, Langmuir probes (LP) provide information on the electron temperature $T_{e}$, ion current density $j_{\text {sat }}$, Mach number, turbulent transport, etc. However, other important parameters such as the ion and electron heat transmission coefficients $\gamma_{i, e}$, the ion sound speed $c_{s}$, the electron density $\mathrm{n}_{\mathrm{e}}$ or the ion pressure $\mathrm{p}_{\mathrm{i}}$ and the sputtering rates, depend also on the ion temperature $\mathrm{T}_{\mathrm{i}}$, which can not be measured using LPs.

Significant effort has been invested to measure SOL $\mathrm{T}_{\mathrm{i}}$. Though sporadic and often subject to large uncertainty, such measurements have demonstrated that $T_{i}>T_{e}$ in the SOL as well as in the edge of the confined plasma (referred to as "edge"), except in high ion-electron collisionality regime.

In contrast to the measurements, it is frequently assumed that " $\mathrm{T}_{\mathrm{i}}=\mathrm{T}_{\mathrm{e}}$ in the SOL" by the plasma boundary community. Although the disagreement of this assumption with the measurements is often recalled in the literature, it is rarely demonstrated that equipartition is to be expected in a given plasma parameter regime. Similarly, in low-to-moderate ion-electron collisionality regimes, where $T_{i}>T_{e}$, a discussion of the sensitivity of the results to $T_{i} / T_{e}$ is often lacking.

Most measurements of SOL ion energies were performed in L-mode discharges, and only very limited data exist on the ion energies in the far SOL (i.e. $\sim 2-3$ characteristic SOL power widths outside the separatrix) during edge localized mode (ELM) instabilities and between ELMs. Such information would be of a large assistance for validating the assumptions used for 
the ITER burning plasma heat load specifications, which in turn, determine the design of the ITER blanket module shaping and power handling capacity. Power balance analysis in the ASDEX Upgrade (AUG) tokamak revealed that $\sim 25 \%$ of the plasma energy loss by ELMs is deposited on non-divertor components [1], providing evidence that ELM ions can reach the first wall with a significant fraction of their initial energies. ELM ion impact energies in the far SOL, measured directly for the first time in JET [2], have found agreement with a parallel loss model for ELM filament propagation across the SOL, which predicts for ITER average ion impact energies a factor of $\sim 4$ higher than the physical sputtering threshold of tungsten by deuterium ions [3]. Similar measurements were recently performed in MAST [4] and AUG [5].

The focus of the present review is on the measurements of ion energies in the tokamak plasma boundary in L-mode discharges and during ELMs. Throughout this paper the energies and temperatures are given in $\mathrm{eV}$ and the indication "parallel" or "perpendicular" relates to the magnetic field vector $\mathbf{B}$.

\section{Diagnostics for SOL $T_{i}$ measurements}

Diverse techniques for SOL $T_{i}$ measurements have been developed and reviewed [6-8] in the past and sporadically employed in tokamaks. None is without drawbacks and some remain controversial. Since a full, proper, critical review of the various methods for measuring SOL $T_{i}$ is beyond the scope of this paper, we simply reference them for completeness in Table 1, without discussing their validity.

The only diagnostic we briefly address in this section is a retarding field analyzer (RFA). Though inherently limited in temporal resolution, because of the technical limitations in the voltage sweeping frequencies, a RFA provides SOL $T_{i}$ with a good energy and spatial resolution 
for most plasma parameter regimes. Its use in a number of tokamaks [2, 4-5, 9-36], extensive instrumental study [10, 12-13 16, 22, 27, 34, 36-39], and the straightforward data interpretation makes a RFA one of the only widely accepted diagnostics for SOL $\mathrm{T}_{\mathrm{i}}$ measurements. In general, a RFA consists of two grids and a collector, separated from the plasma by a slit plate. The analyzer is aligned with $\mathbf{B}$. A fraction of the incident ion flux is transmitted through the aperture cut in the slit plate. The slit plate also repels most electrons by means of an externally applied negative voltage. By sweeping the positive voltage applied to one of the grids $\left(\mathrm{V}_{\text {grid }}\right)$, the ion current-voltage characteristic is measured by the collector. $T_{i}$ is obtained from the slope of the collector current plotted against $\mathrm{V}_{\text {grid }}$, assuming that the ion distribution approximates a Maxwellian, at least in the high energy tail (this is likely to be a good approximation at least when the ion source is distributed over a region long compared to the ion collisional mean free path, Fig. 25.1 in [40]). Large constant negative voltage is applied to the second grid in order to repel the electrons able to surmount the slit plate voltage, or to suppress any secondary electrons emitted inside the analyzer by ion impact. Alternative grid configurations and bias schemes have been used for RFAs $[7,10,12-13,15,22,26,30] . \mathrm{T}_{\mathrm{e}}$ can also be measured by a RFA, either simultaneously with $\mathrm{T}_{\mathrm{i}}$, using the slit plate as a simple Langmuir probe [28-29, 32-36], or by means of reversed grids polarity $[9,12-13,16,18,30]$.

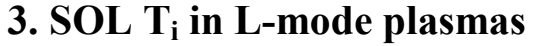

\section{$3.1 T_{i} / T_{e}$ from the edge of the confined plasma into the SOL}

The compilation of the ion-to-electron temperature ratios from Table 1 is plotted in Fig. 1. Measurements for which the radial position was not indicated in the references are omitted. For 
the sake of clarity, the "extreme" $T_{i} / T_{e}>11[6,46]$ are also not plotted. Measurements were obtained in limited $[11,18,26,29-30,36,42-43,48,51-54,57-59]$ as well as diverted $[5,6,25$, $46,49,55]$ L-mode discharges. Vertical lines indicate the range of $T_{i} / T_{e}$ in the plasma density scans at fixed radius $[16,18,29,36,53-54]$. Variations of the main plasma parameters and geometry, the poloidal location of the measurement and differences in experimental techniques may account for the large scatter of $T_{i} / T_{e}$.

While the lower limit of $T_{i} / T_{e}(\sim 1)$ stays almost constant from the edge into the SOL, the upper limit increases strongly with radius and is significantly higher than one at the last closed flux surface (LCFS) and in the SOL. Clearly, there is no "typical" value of $T_{i} / T_{e}$ in the tokamak plasma boundary.

In both conduction, and sheath-limited SOLs, which are characteristic of divertor and limiter configurations respectively, the trends seen in Fig. 1 can be explained without resorting to extreme assumptions about the plasma parameters. Although there may be conditions when other sources and sinks such as charge-exchange collisions, radiation or cross-field conduction may play a role, it is a fairly safe generalization that parallel conduction $\kappa T^{5 / 2} \nabla_{/ /}$dominates the power balance in the conduction-limited SOL. The ion parallel heat conductivities is much smaller than that of the electrons $\left(\kappa_{\mathrm{i}}<<\kappa_{\mathrm{e}}\right)$, which leads to $\mathrm{T}_{\mathrm{i}} / \mathrm{T}_{\mathrm{e}} \approx 4$ just outside the separatrix, assuming thermally decoupled deuterium ions and electrons. The increase of the ionelectron thermal coupling (e.g. by increasing the plasma density $n_{e}$ ) tends to make both temperatures converge. Thermal equilibrium is expected when the parallel ion transit time along the SOL $\tau_{/ /} \propto \mathrm{L}_{\text {con }} / \sqrt{\mathrm{T}_{\mathrm{i}}+\mathrm{T}_{\mathrm{e}}}$ (with $\mathrm{L}_{\text {con }}$ being the parallel connection length) becomes substantially longer than the ion-electron thermalization time $\tau_{\mathrm{ie}} \propto \mathrm{T}_{\mathrm{e}}^{3 / 2} / \mathrm{n}_{\mathrm{e}}$, Sec. 3.2. Depending 
on the plasma parameters regime, we may thus anticipate $T_{i} / T_{e} \approx 1-4$ just outside the separatrix.

In Tore Supra it is observed experimentally that while the SOL $\mathrm{T}_{\mathrm{i}}$ varies by more than an order of magnitude, tracking the variation of the core parameters rather closely, SOL $\mathrm{T}_{\mathrm{e}}$ hardly changes at all and seems to be decoupled from the core plasma, Sec. 3.2. Since parallel conduction typically dominates the electron power balance (from the simple two-point model $\mathrm{T}_{\mathrm{e}} \propto \mathrm{Q}_{\mathrm{e}}^{2 / 7}[40]$ ), a large variation of the heat conducted across the LCFS $\mathrm{Q}_{\mathrm{e}}$ has only a weak effect on SOL $T_{e}$. On the other hand, since $\kappa_{i}<<\kappa_{e}$, the ions remain coupled to the core plasma. It is also because of $\kappa_{\mathrm{i}}<<\kappa_{\mathrm{e}}$ that $\mathrm{T}_{\mathrm{e}}$ usually drops faster than $\mathrm{T}_{\mathrm{i}}$ in the SOL.

In the sheath-limited SOL, the Debye sheath generally removes more heat from the electrons compared to ions so that $T_{i} / T_{e}$ increases with radius in the SOL [40,60]. As in the conduction-limited SOL, strong thermal coupling of ions and electrons can restore the equipartition.

In the edge plasma, large temperature gradients make a difference of SOL temperatures of the order of tens of electron volts unimportant so that $T_{i} / T_{e}$ quickly converges to unity. The somewhat steeper profiles of $T_{e}$ compared to $T_{i}$, which are typically observed in the edge, could be explained by a larger radial transport of ion heat compared to electron heat. This is an assumption often required in the transport codes to match the measured edge temperatures, e.g. $[50]$. 


\subsection{Variation of SOL $T_{i}$ and $T_{e}$ with the global plasma parameters}

The compilation in Fig. 1, which includes measurements performed under different plasma conditions, does not allow an identification of the influence of individual macroscopic plasma parameters on $T_{i} / T_{e}$. Some trends were investigated in the past by varying the selected plasma parameter and keeping other parameters constant.

Fig. 2 illustrates the variation of the LCFS $T_{i}$ and $T_{e}$ with $n_{e}$ measured in $\mathrm{D}^{+}$plasma in Tore Supra using a RFA. Both $T_{i}$ and $T_{e}$ decrease with $n_{e}$, roughly following pressure conservation. $T_{i}$ decreases an order of magnitude, whilst $T_{e}$ is much less affected. As a consequence, $T_{i} / T_{e}$ drops from $3 \rightarrow 1$ in going from lowest to highest density. Such observations are not unique to Tore Supra and have been previously reported on other tokamaks (e.g. $[16,18,48,52-54])$ and reproduced by modeling [61]. Here, as well as in the earlier studies $[16,18,54]$, the drop of $T_{i} / T_{e}$ with increasing $n_{e}$ is consistent with the increase of the ionelectron thermal coupling, Fig. 2.

In common with the effect observed with $\mathrm{n}_{\mathrm{e}}$, additional heating generally has a more marked effect on $T_{i}$ than $T_{e}$. This leads to generally higher $T_{i} / T_{e}$ in additionally heated plasmas compared to ohmic plasmas, irrespective of whether the heating is applied to core ions or electrons. In DITE, the injection of $140 \mathrm{~kW}$ of electron cyclotron resonance heating power (corresponding to $\approx 1 / 2$ of the ohmic power $\mathrm{P}_{\text {ohm }}$ ) was associated with an increase of SOL $\mathrm{T}_{\mathrm{i}}$ and $\mathrm{T}_{\mathrm{e}}$ by a factor of 3 and 2 , respectively [16]. The effect of the additional heating was, surprisingly, more marked at larger distances from the LCFS. Little effect on the edge temperatures was observed for lower-hybrid $(\mathrm{LH})$ heated discharges with $\mathrm{P}_{\mathrm{LH}} \approx 1 / 2 \mathrm{P}_{\text {ohm }}$ [16]. A factor of $\sim 2$ increase of $T_{i} / T_{e}$ in TEXTOR discharges with neutral beam injection $\left(P_{N B I}=1 \mathrm{MW}\right)$ in 
addition to $\mathrm{P}_{\text {ohm }}$ compared to ohmic plasmas was explained by preferential ion heating [52]. A similar effect of NBI on $T_{i} / T_{e}$ was measured by the RFA in JET [20]. A factor of $\sim 2-3$ increase of $\mathrm{T}_{\mathrm{i}} / \mathrm{T}_{\mathrm{e}}$ from ohmically- to LH-heated as well as ion-cyclotron-resonance-heated (ICRH) discharges was measured by a RFA in the Tore Supra SOL. In Tore Supra, at approximately constant power in the $\mathrm{SOL} \mathrm{P}_{\mathrm{SOL}}$ (i.e. the total heating power reduced by the radiated power and the ripple losses [62]) both, ICRH and LH heating, leads to very similar SOL temperatures, Fig. 3. This indicates that the higher $T_{i} / T_{e}$ in additionally heated plasmas could be simply associated to weaker ion-electron collisional coupling because of higher temperatures, rather than to the preferential ion heating, as suggested in [52]. $T_{i}$ and $T_{e}$ independent of the heating method for fixed $\mathrm{P}_{\mathrm{SOL}}$ and $\mathrm{n}_{\mathrm{e}}$ can be explained by simple power balance arguments. The fact that, in contrast to [16], the SOL temperatures in Tore Supra are higher in LH- compared to ohmically-heated plasmas might be explained by significantly larger $\mathrm{P}_{\mathrm{LH}} / \mathrm{P}_{\mathrm{ohm}}$ compared to the experiments in DITE [16].

Simultaneous increase of $T_{i}$ and $T_{e}$ near the LCFS due to the degraded thermal insulation in the edge plasmas induced by the growth of a MHD mode was reported in [16]. Very few studies address the influence of other plasma parameters such as the toroidal magnetic field [32] or the radiated power fraction [34] on $T_{i} / T_{e}$.

\subsection{Radial dependence of $S O L T_{i}$ and $T_{e}$}

Although the simultaneous measurements of the SOL ion and electron temperature efolding lengths, $\lambda_{\text {Ti,e }}$, are rare, they indicate that $T_{e}$ usually falls somewhat steeper than $T_{i}$, Fig. 4., which is consistent with faster parallel energy losses of electrons in the SOL, Sec. 3.1. In the 
sheath-limited SOL (which may be a good approximation at least for those measurements in Fig. 4 that were obtained in limiter tokamks), $\lambda_{\mathrm{Ti}} / \lambda_{\mathrm{Te}}$ is determined by the value of the electron heat transmission coefficient, electron cross-field heat conduction relative to the heat diffusion and the degree of the ion-electron thermal coupling [60]. We may thus anticipate a broad range of $\lambda_{\mathrm{Ti}} / \lambda_{\mathrm{Te}}$ in the SOL. At the same time, the poloidal asymmetry of the radial energy transport (due to the expulsion of the magnetic-field-aligned filaments on the plasma outboard side) makes $\lambda_{\text {Ti,e }}$ functions of the poloidal angle. Enhanced radial energy transport on the outboard side was demonstrated in the past for electrons (see reference in [35]), and recently also for ions (Fig. 4 and [35]).

\section{ELM ion energies in the far SOL}

First direct measurements of the far SOL ELM ion impact energies were obtained in JET using a RFA [2]. The probe was inserted from the top of the plasma into a lower single null (LSN) type-I ELMy H-mode discharge. The ion repelling grid was biased to $\mathrm{V}_{\text {grid }} \cong 400 \mathrm{~V}$ (we recall, Sec. 2, that only ions with parallel impact energies $E_{i / /} \geq e Z_{i} V_{\text {grid }}$ can reach the RFA collector). The probe distance from the separatrix $\Delta \mathrm{r}_{\text {sep }}>4 \mathrm{~cm}$. The response of the RFA collector to ELMs, seen as sharp bursts of collector current $I_{c}$ (in contrast to $I_{c} \approx 0$ during interELM periods), indicated the presence of ELM ions with $E_{i / /} \geq 400 \mathrm{eV}$ in the far SOL. A fluid filament loss model [3] applied to the JET experiment [2] predicts the ELM ion temperature $\mathrm{T}_{\mathrm{iELM}}=100-150 \mathrm{eV}$ and $\mathrm{T}_{\mathrm{iELM}}=2-2.5 \mathrm{~T}_{\mathrm{eELM}}$ in the far SOL. The model was successful in

reproducing the measured RFA collector currents [2-3]. $\mathrm{T}_{\mathrm{iELM}}>\mathrm{T}_{\mathrm{eELM}}$ because of higher parallel electron losses has been observed also in kinetic simulations (e.g. [63]). 
$\mathrm{T}_{\mathrm{iELM}} \cong 30-60 \mathrm{eV} \approx 1-2 \mathrm{~T}_{\text {eELM }}$ (with $\mathrm{T}_{\text {eELM }}$ reported in [64]) for $4-8 \mathrm{~cm}$ outside the outer midplane separatrix has been obtained from the comparison of the e-folding lengths of $j_{\text {sat }}$ measured by the LP and the heat flux density measured by an IR camera in AUG [45].

Recently, measurements of the ELM ion energies in the far SOL using RFAs were performed in MAST [4] and AUG [5]. In both cases, the RFA response to ELMs is similar to what was observed in JET [2].

In MAST, the ELM ion energies were studied by a RFA in LSN type-I and double null (DN) type-III ELMy H-mode discharges with $\mathrm{P}_{\mathrm{NBI}}=1.3-3.3 \mathrm{MW}$. The RFA was located at the outer midplane. In the LSN discharges, characterized by pedestal temperatures $T_{i p e d}=400 \mathrm{eV}$ and $T_{\text {eped }}=300 \mathrm{eV}$, bursts of $I_{c}$ synchronous with ELMs have been measured at fixed $\mathrm{V}_{\text {grid }}=500 \mathrm{~V}$ and up to $\Delta \mathrm{r}_{\text {sep }}=20 \mathrm{~cm}$. Large radial extension of the ELM filaments in the LSN discharges could be explained by the fact that these type-I ELMs were triggered by the sawtooth instability, carrying particles from the hot core plasma and producing energetic ELMs. In contrast to the LSN discharges, in the DN configuration $\left(T_{\text {ped }}=110 \mathrm{eV}\right)$ no $I_{c}$ has been detected during type-III ELMs for $\Delta \mathrm{r}_{\text {sep }}>10 \mathrm{~cm}$ and $\mathrm{V}_{\text {grid }}=200 \mathrm{~V}$.

In AUG, the RFA measurements were performed in LSN H-mode discharges with $\mathrm{P}_{\mathrm{NBI}}=2.5 \mathrm{MW}$. The RFA was mounted on the reciprocating drive located $31 \mathrm{~cm}$ above the outboard midplane. Similar to the RFA measurements in JET and MAST, large bursts of the collector current were synchronous with ELMs for $\mathrm{V}_{\text {grid }} \cong 30-160 \mathrm{~V}$. Evidence for the filamentary structure of ELMs was observed on the time traces of collector current $\mathrm{I}_{\mathrm{cELM}}$ as in [2]. The duration of and the time interval between individual ion current filaments (both 
$100-200 \mu \mathrm{s}$ ) was found to be consistent with earlier LP measurements in AUG [45]. $\mathrm{I}_{\mathrm{cELM}}$ decreases with increasing $\mathrm{V}_{\text {grid }}$ as well as increasing $\Delta \mathrm{r}_{\text {sep }}$. Almost no $\mathrm{I}_{\mathrm{cELM}}$ was detected in the shadow of the outboard limiter, most likely due to enhanced parallel losses because of shorter $\mathrm{L}_{\text {con }}$. Similarly, $\mathrm{I}_{\mathrm{c}}$ was almost zero during inter-ELM periods except for $\mathrm{V}_{\text {grid }}<50 \mathrm{~V}$. Far SOL inter-ELM $\mathrm{T}_{\mathrm{i}} \approx 12 \mathrm{eV} \approx \mathrm{T}_{\mathrm{e}}$ (consistent with a strong ion-electron thermal coupling) has been estimated from the slope of inter-ELM $I_{c}$ plotted against $V_{\text {grid }}$ [5]. The ELM ion temperature $\mathrm{T}_{\mathrm{iELM}} \approx 50-90 \mathrm{eV}$ has been estimated from the comparison of the collector and the slit plate ion currents measured at $\Delta \mathrm{r}_{\text {sep }} \cong 6 \mathrm{~cm}$ (the large range in $\mathrm{T}_{\mathrm{iELM}}$ is mainly due to the uncertainty in the ion current transmission factor of the analyzer [5]). A similar range of $\mathrm{T}_{\mathrm{iELM}}$ in the far SOL was predicted from Monte Carlo simulations of ELM filament propagation across the SOL [65] as well as the filament loss model [3], Fig. 5, assuming a mean radial filament velocity $\mathrm{v}_{\mathrm{r}}=1.5 \mathrm{~km} \mathrm{~s}^{-1}$ typically measured in the far SOL of AUG [65-66].

Close proximity of the simulated and the measured ELM ion energies in the far SOL of JET and AUG increase confidence in model predictions of ELM-wall interactions in ITER. The filament loss model predicts $\mathrm{T}_{\mathrm{iELM}} \cong 260-420 \mathrm{eV}$ (similar to $\mathrm{T}_{\mathrm{iELM}} \cong 200-500 \mathrm{eV}$ obtained from the Monte Carlo simulations [65]) and $\mathrm{T}_{\mathrm{eELM}} \cong 100-200 \mathrm{eV}$ at the wall radius in ITER, implying the average ELM ion impact energy $\approx 3 \mathrm{~T}_{\mathrm{eELM}}+2 \mathrm{~T}_{\mathrm{iELM}}=0.8-1.4 \mathrm{keV}$, which is a factor or $\sim 4$ higher than the sputtering threshold of tungsten [3]. Large confidence intervals of $\mathrm{T}_{\mathrm{iELM}}$ from the models are mainly due to the uncertainty in $\mathrm{v}_{\mathrm{r}}$. 


\section{Summary}

Higher mobility of electrons compared to ions results in $T_{i}>T_{e}$ in the sheath- and conduction-limited SOL. Strong ion-electron collisional coupling is needed to produce thermal equilibrium.

$\mathrm{T}_{\mathrm{i}}>\mathrm{T}_{\mathrm{e}}$ in the SOL has been observed in a number of limiter and divertor tokamaks. The large spread in measured $T_{i} / T_{e}$ values $\left(T_{i} / T_{e}=1-10 \mathrm{~cm}\right.$ outside the LCFS $)$ has important consequences for the estimation of some edge parameters from LP measurements in the main SOL. Assuming $T_{i}=T_{e}$ can lead to the underestimation of $\gamma=\gamma_{i}+\gamma_{e}$ and $p_{i}$ by up to a factor 4, and $\mathrm{c}_{\mathrm{s}}$ by up to a factor of 2.5 , or the overestimation of $\mathrm{n}_{\mathrm{e}}$ by more than a factor of 2 . The same assumption can also significantly underestimate the sputtering rate $\left(\approx \mathrm{eZ}_{\mathrm{i}} \mathrm{V}_{\text {sheath }}+2 \mathrm{~T}_{\mathrm{i}}, \mathrm{V}_{\text {sheath }}\right.$ being the sheath potential) from non-divertor components, such as the antenna limiters, especially by low-Z impurities. Additionally, since $T_{e}$ typically decreases faster with radius than $T_{i}$, the assumption of constant $T_{i} / T_{e}$ across the SOL may also influence the analysis of the radial variation of the SOL parameters that are a function of $T_{i} / T_{e}$.

Since $T_{i} / T_{e}$ varies strongly with the macroscopic parameters such as the plasma density or heating power, there is no "typical" value of $T_{i} / T_{e}$ in the SOL or at the LCFS. Measurements of SOL $\mathrm{T}_{\mathrm{i}}$ are, therefore, needed for every particular combination of plasma parameters. Though sporadic and often subject to large uncertainty, such measurements are not that difficult (3220 RFA reciprocations within its six years operation in the Tore Supra tokamak is an outstanding example [34]) and should be performed more systematically. On the other hand, $T_{i}$ is practically unknown on the turbulence timescale, as new diagnostics, such as the segmented tunnel probe [48], are not yet sufficiently mature to provide reliable data. 
Given the importance of the ELM-wall interaction in ITER regarding the sputtering of impurities, large effort has recently been invested to study the ELM ion energies $E_{\mathrm{iELM}}$ in the far SOL. First direct measurements of $\mathrm{E}_{\mathrm{iELM}}$ were obtained in JET, and more recently in MAST and AUG, using RFAs. These measurements have consistently found agreement with the state-of-art models of the ELM transients [3,65], increasing confidence in their predictive capability towards ITER. The situation is, however, far from being ideal, as the study of the far SOL ELM ion energies is in its infancy and only a very limited data set exists. Faced with the lack of adequate

measurements, $T_{\mathrm{iELM}}$ is estimated using simplifying assumptions and thus subject to large errors. There is also a substantial uncertainty in the predictions of the models, since it is not known where between the pedestal and the separatrix the filaments start to lose their particles and energy and what determines the velocity of their propagation across the SOL.

\section{Acknowledgements}

The authors acknowledge the helpful comments from D. Coster, P. C. Stangeby and D. Tskhakaya.

\section{References}

[1] A. Herrmann et al., Plasma Phys. Control. Fusion 46 (2004) 971.

[2] R. A. Pitts et al., Nucl. Fusion 46 (2006) 82.

[3] W. Fundamenski et al. Plasma Phys Control Fusion 48 (2006) 109.

[4] P. Tamain et al., these Proceedings, paper P2-94.

[5] M. Kočan et al., in preparation.

[6] P. Staib, J. Nucl. Mater. 111-112 (1982) 109. 
[7] G. F. Matthews, Plasma Phys. Control. Fusion 36 (1994) 1595.

[8] V. I. Demidov et al., Rev. Sci. Instrum. 73 (2002) 3409.

[9] H. Kimura et al., Nucl. Fusion 18 (1978) 1195.

[10] H. Kimura et al., Japan. J. Appl. Phys. 18 (1979) 2275.

[11] M. El Shaer, Ph D Thesis, Scientific and Medical University, Grenoble, 1981.

[12] G. F. Matthews, J. Phys. D: Appl. Phys. 17 (1984) 2243.

[13] A. S. Wan et al., Rev. Sci. Instrum. 57 (1986) 1542.

[14] R. A. Pitts, J. Nucl. Mater. 162-164 (1989) 568.

[15] R. A. Pitts and G. F. Matthews, J. Nucl. Mater. 176-177 (1990) 877.

[16] R. A. Pitts, Ph D Thesis, University of London, 1991.

[17] R. A. Pitts, Phys. Fluids B 3 (1991) 2871.

[18] G. F. Matthews, Nucl. Fusion 31 (1991) 1495.

[19] R. A. Pitts, Contrib. Plasma Phys. 36S (1996) 87.

[20] H. Y. Guo et al., Contrib. Plasma Phys. 36 (1996) 81.

[21] S. Raychaudhuri, Contrib. Plasma Phys. 39 (1999) 359.

[22] R. Nachtrieb, Ph D Thesis, Massachusetts Institute of Technology, 2000.

[23] R. Nachtrieb et al., Rev. Sci. Intrum. 71 (2000) 4107.

[24] R. A. Pitts et al., Rev. Sci. Instrum. 74 (2003) 4644.

[25] R. A. Pitts et al., Proc. $30^{\text {th }}$ EPS Conference on Controlled Fusion and Plasma Physics St.

Petersburg, 7-11 July 2003 ECA Vol. 27A, P-2.84.

[26] I. Nedzelskiy et al., Rev. Sci. Instrum. 77 (2006) 10E729-1.

[27] M. Kočan et al., Rev. Sci. Instrum. 79 (2008) 073502.

[28] M. Kočan et al., Proc. 35th EPS Conf. on Plasma Phys. Hersonissos, 9 - 13 June 2008 ECA

Vol.32D, P-1.006 (2008). 
[29] M. Kočan et al., Plasma Phys. Control. Fusion 50 (2008) 125009.

[30] M. Dreval et al., Rev. Sci. Instrum. 80 (2009) 103505.

[31] J. P. Gunn et al., J. Nucl. Mater. 390-391 (2009) 904.

[32] M. Kočan et al., J. Nucl. Mater. 390-391 (2009) 1074.

[33] M. Kočan and J. P. Gunn, Proc. 36th EPS Conf. on Plasma Phys. Sofia, 29 June - 3 July 2009 ECA Vol.33E, P-4.203 (2009).

[34] M. Kočan, Ph D Thesis, University of Nancy, 2009.

[35] M. Kočan and J. P. Gunn, Plasma Phys. Control. Fusion 52 (2010) 045010.

[36] M. Kočan et al., to be published in the Contrib. Plasma Phys., 2010.

[37] A. W. Molvik, Rev. Sci. Instrum. 52 (1981) 704.

[38] G. F. Matthews, Ph D Thesis, University of Oxford, 1984.

[39] A. S. Wan, Ph D Thesis, Massachusetts Institute of Technology, 1986.

[40] P. C. Stangeby, The Plasma Boundary in magnetic Fusion Devices (Bristol: Institute of Physical Publishing) 2000.

[41] W. R. Wampler et al., J. Vac. Sci. Technol. A 1 (1983) 827.

[42] J. Adamek et al., Contrib. Plasma Phys. 48 (2008) 1.

[43] P. C. Stangeby et al., J. Vac. Sci. Technol. A 1 (1983) 1302.

[44] T. Lunt et al., Plasma Phys. Control. Fusion 49 (2007) 1783.

[45] A. Herrmann et al., J. Nucl. Mater. 363-365 (2007) 528.

[46] K. Uehara et al., Nucl. Fusion 38 (1998) 1665.

[47] K. Hötker and H.-J. Belitz, Rev. Sci. Instrum. 61 (1990) 114.

[48] M. Kočan et al., J. Nucl. Mater. 363-365 (2007) 1436.

[49] M. Reich et al., Plasma Phys. Control. Fusion 46 (2004) 797.

[50] M. Reich, Ph D Thesis, Ludwig-Maximilians-Universität München, 2004. 
[51] R. P. Schorn et al., Nucl. Fusion 32 (1992) 351.

[52] A. Huber et al., Plasma Phys. Control. Fusion 42 (2000) 569.

[53] A. Kreter, Ph D Thesis, Düsseldorf University, 2001.

[54] P. Bogen et al., J. Nucl. Mater. 220-222 (1995) 472.

[55] N. Asakura et al., J. Nucl. Mater. 241-243 (1997) 559.

[56] G. Staudenmaier et al., J. Nucl. Mater. 93-94 (1980) 121.

[57] S. K. Erents and P. C. Stangeby, J. Nucl. Mater. 111-112 (1982) 165.

[58] S. A. Cohen et al., J. Nucl. Mater. 93-94 (1980) 109.

[59] W. R. Wampler et al., J. Nucl. Mater. 102 (1981) 304

[60] P. C. Stangeby and G. M. Cracken, Nucl. Fusion 30 (1990) 1225.

[61] R. Zagorski et al., Contrib. Plasma Phys. 34 (1994) 466.

[62] V. Basiuk et al., Nucl. Fusion 41 (2001) 477.

[63] R.A. Pitts et al., Nucl. Fusion 47 (2007) 1437.

[64] H. W. Müller et al., Towards fast electron temperature measurements in the SOL of ASDEX Upgrade using swept Langmuir probes, to be published in Contrib. Plasma Phys., 2010.

[65] A. Kirk et al., J. Nucl. Mater. 390-391 (2009) 727.

[66] A. Schmid et al., Plasma Phys. Control. Fusion 50 (2008) 045007 


\section{Figure / table captions}

Table 1. Survey of techniques for SOL $T_{i}$ measurements. $T_{i} / T_{e}$ indicates the approximate value of the measured SOL ion-to-electron temperature ratio (not available if the characteristic $\mathrm{T}_{\mathrm{e}}$ value was not stated in the reference). (a) ELM $T_{i} / T_{e}$. (b) $T_{i} / T_{e}$ for the cold majority / hot minority ions.

Figure 1. Ion-to-electron temperature ratios measured in the plasma boundary of different tokamaks, plotted against the distance from the plasma center normalized to minor radius. Lined data points correspond to $\mathrm{T}_{\mathrm{i}} / \mathrm{T}_{\mathrm{e}}$ profiles.

Figure 2. Ion and electron temperatures measured by RFA at the LCFS in the ohmic density scan in Tore Supra. Temperatures are plotted against the volume-averaged plasma density. The ratio of the parallel ion transit time through the SOL to the ion-electron thermalization time evaluated 2-3 $\mathrm{cm}$ outside the LCFS indicates the degree of the ion-electron collisional coupling. Full symbols: detached discharges.

Figure 3. Top: Power balance in the Tore Supra discharge \#39539. The power carried across the LCFS by thermal particles $\mathrm{P}_{\mathrm{SOL}}$ (i.e. the total heating power reduced by the radiated power and the ripple losses), is roughly constant during the discharge. RFA reciprocations are indicated by arrows. Bottom: SOL $\mathrm{T}_{\mathrm{i}}$ and $\mathrm{T}_{\mathrm{e}}$ measured by RFA (symbols correspond to individual reciprocations). Volume-averaged plasma density $\left\langle\mathrm{n}_{\mathrm{e}}\right\rangle=2.3-2.5 \times 10^{19} \mathrm{~m}^{-3}$. 
Figure 4. Ion and electron temperature e-folding lengths measured in the SOL of different tokamaks. Inboard / outboard indicates the plasma contact point.

Figure 5. Far SOL ELM ion temperature $T_{\text {iELM }}$ estimated from the RFA measurements in AUG. $\mathrm{T}_{\mathrm{iELM}}$ from RFA is compared with the predictions from the Monte Carlo simulations of the ELM filaments propagation across the SOL (full) [65] and the fluid filament loss model [3] assuming two different birth locations of the ELM filaments: pedestal top (dotted), separatrix (dashed). The simulations were performed assuming the characteristic radial filament velocity $\mathrm{v}_{\mathrm{r}}=1.5 \mathrm{kms}^{-1}$ [65-66]. 


\section{Table 1}

\begin{tabular}{|c|c|c|c|}
\hline Technique & Tokamak & $T_{\mathrm{i}} / T_{\mathrm{e}}$ & Reference \\
\hline \multirow[t]{2}{*}{ Carbon resistance probe } & PLT & & [41] \\
\hline & PDX & & [41] \\
\hline \multirow[t]{2}{*}{$\mathbf{E} \times \mathbf{B}$ analyzer } & ASDEX & $2-16$ & [6] \\
\hline & DITE & 1 & [12] \\
\hline \multirow[t]{2}{*}{ Katsumata probe } & CASTOR & $1-2$ & [42] \\
\hline & Petula & $0.6-0.8$ & [11] \\
\hline LP + thermocouple & DITE & $2-7$ & [43] \\
\hline $\mathrm{LP}+$ force sensor & ISTTOK & $0.4-2$ & [44] \\
\hline LP + IR camera & AUG & $1-2^{(a)}$ & {$[45]$} \\
\hline Plasma ion mass spectrometer & DITE & $3-8$ & [18] \\
\hline \multirow[t]{12}{*}{ Retarding field analyzer } & Alcator C & $2-7$ & [13] \\
\hline & C-Mod & $1(5)^{(\mathrm{b})}$ & {$[22]$} \\
\hline & AUG & 1 & [5] \\
\hline & DITE & $3-8$ & {$[16,18]$} \\
\hline & DIVA & $2-3$ & {$[10]$} \\
\hline & ISTTOK & $1-2$ & [26] \\
\hline & JET & $1-3$ & {$[20,24-25]$} \\
\hline & MAST & $1-2$ & {$[4]$} \\
\hline & Petula & 2 & [11] \\
\hline & SINP & 1 & [21] \\
\hline & STOR-M & $1-3$ & [30] \\
\hline & Tore Supra & $1-8$ & {$[27-29,32-36]$} \\
\hline \multirow[t]{2}{*}{ Rotating double probe } & JFT-2M & $5-26$ & [46] \\
\hline & TEXTOR & 9 & [47] \\
\hline Segmented tunnel probe & CASTOR & $1-3$ & [48] \\
\hline \multirow[t]{4}{*}{ CXRS } & AUG & 2 & {$[49-50]$} \\
\hline & DIVA & $2-3$ & {$[10]$} \\
\hline & TEXTOR & $1-4$ & [51-54] \\
\hline & JT-60U & $2-5$ & {$[55]$} \\
\hline \multirow[t]{2}{*}{ Surface collection probe } & DITE & 2 & [14] \\
\hline & TFR-600 & 10 & {$[56]$} \\
\hline \multirow[t]{2}{*}{ Thermal desorption probe } & DITE & $1-2$ & [57] \\
\hline & PLT & $6-9$ & {$[6,58-59]$} \\
\hline
\end{tabular}


Figure 1

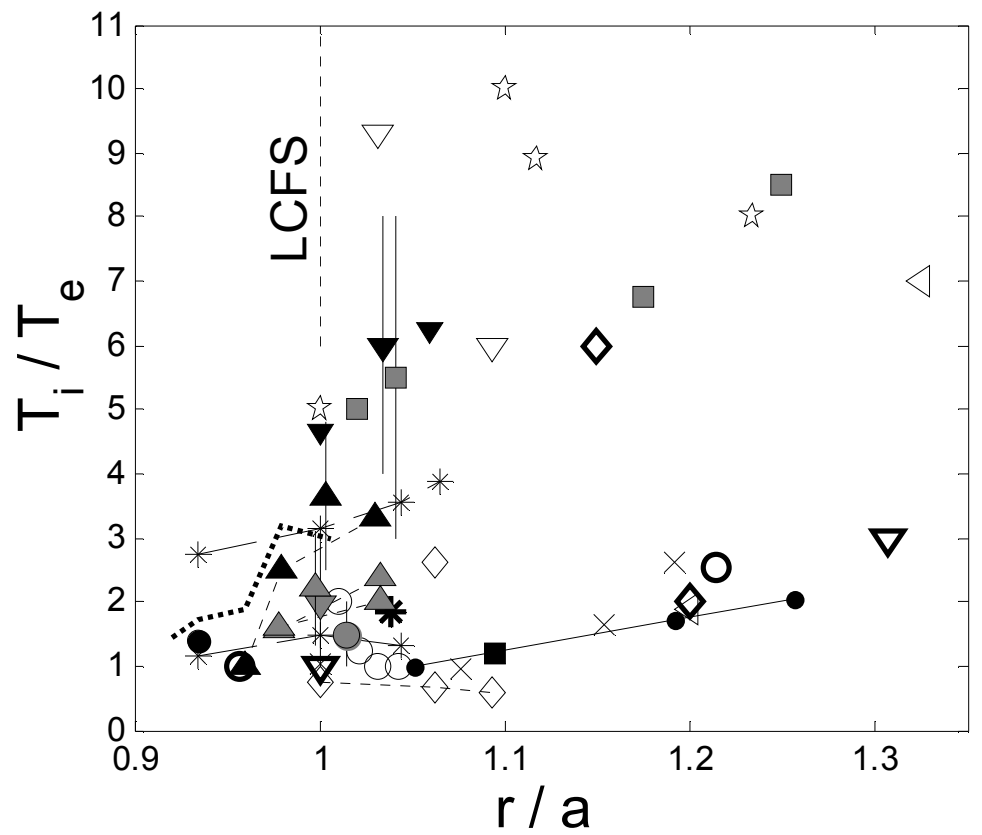

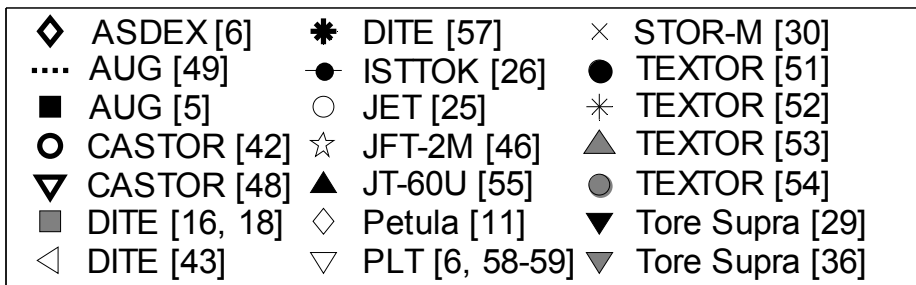


Figure 2

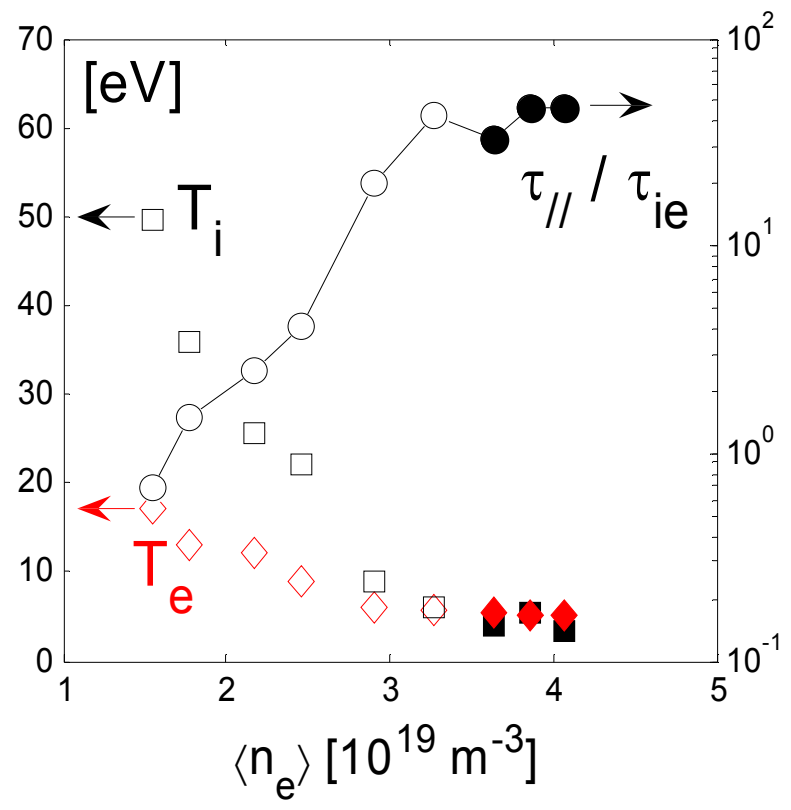


Figure 3
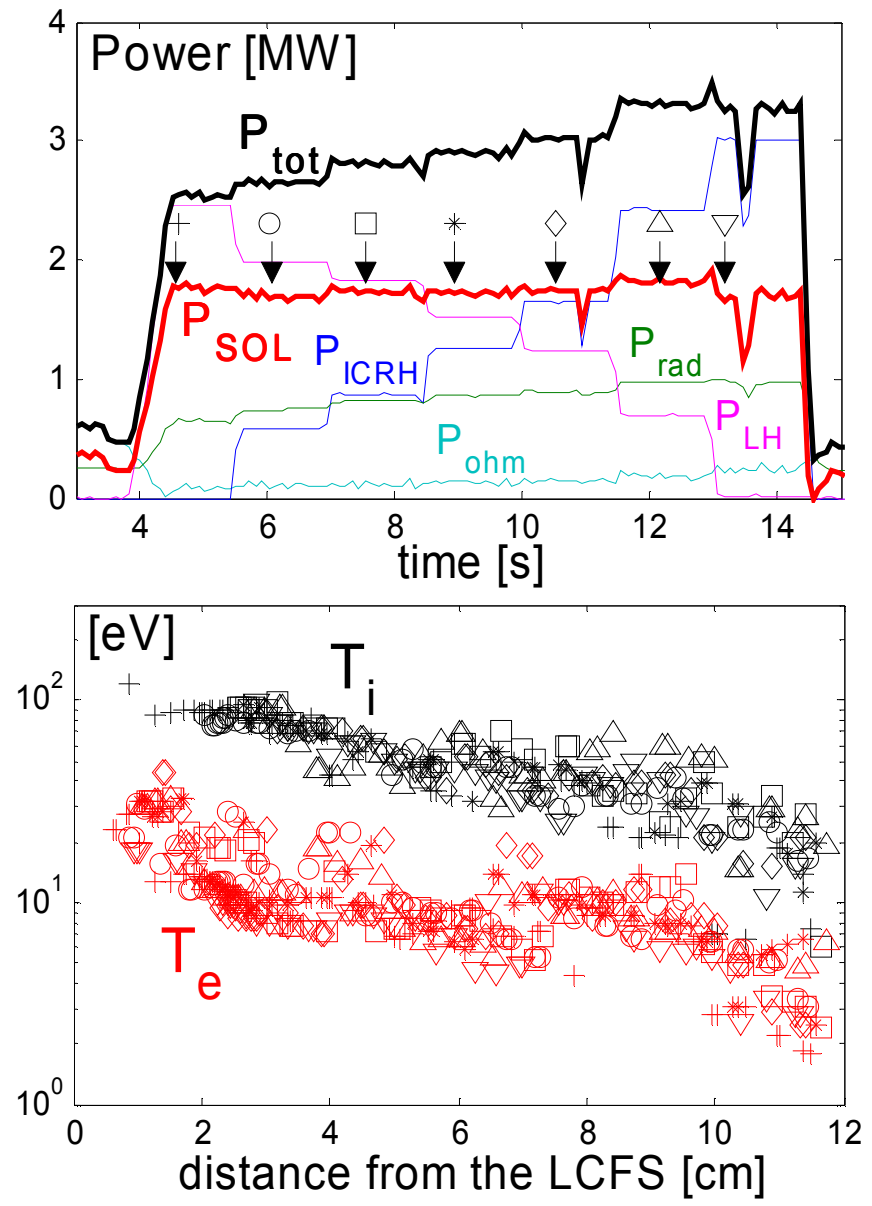
Figure 4

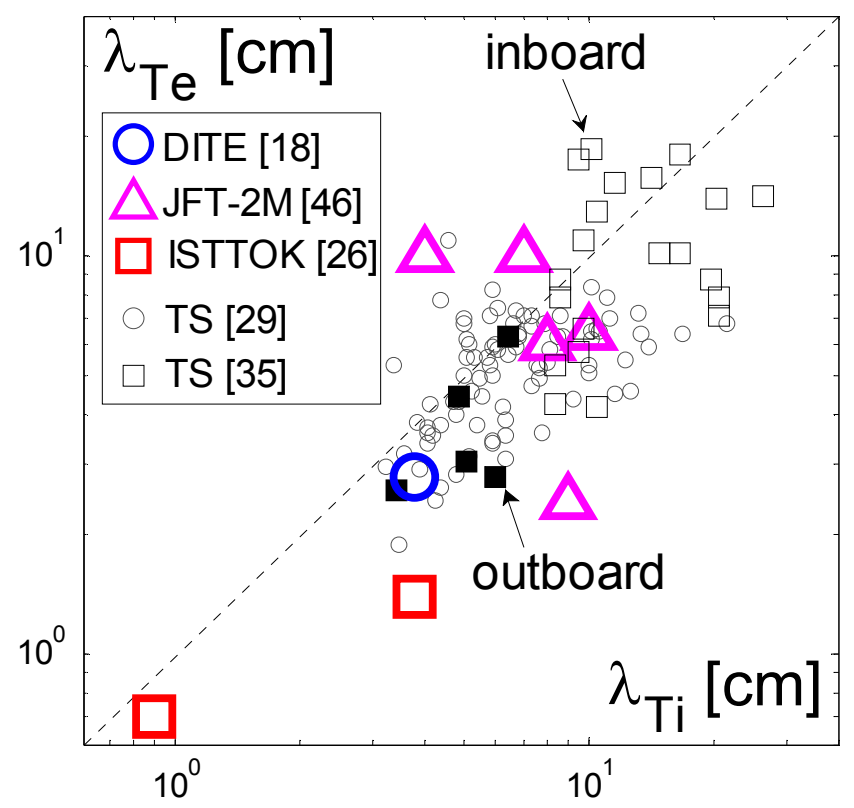


Figure 5

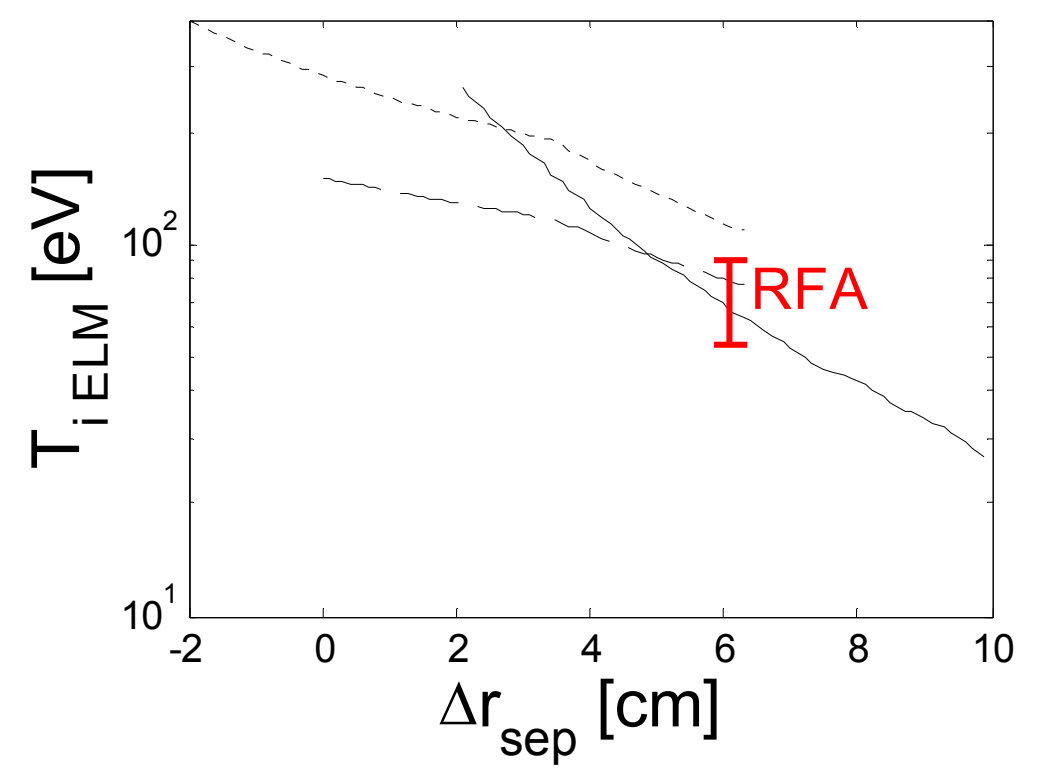

